\title{
Study of Central Regions of AGNs at 6-meter Telescope
}

\author{
V. L. Afanasiev, A. I. Shapovalova, A.N. Burenkov, S. N. Dodonov and \\ V. V. Vlasiouk \\ Special Astrophysical Observatory of Russian Academy of Sciences, \\ Nyzhnij Arkhyz, Russia,357147
}

\begin{abstract}
Kinematic investigations of the central $\left(10^{\prime \prime} \times 10^{\prime \prime}\right)$ regions of the Seyfert galaxies NGC 4151 and Mkn 573 on the 6-m telescope with 3D-spectroscopy methods are presented.
\end{abstract}

\section{Introduction}

Gas emission in permitted and forbidden lines in the central regions $(\approx 1 \mathrm{kpc})$ of Seyfert galaxies is caused, in our opinion, by three subsystems of gas: component \#1, associated with HII regions of the rotating disk; component \#2, associated with the shock waves in the region of the central bar; component \#3, associated with the large-scale Kelvin-Helmholz instability at the boundary of jets observed in these galaxies.

Radial velocities of these subsystems differ by about $150-200 \mathrm{~km} \mathrm{~s}^{-1}$, and the mean velocity dispersion in separate clouds is $30-80 \mathrm{~km} \mathrm{~s}^{-1}$. Positions of gas clouds in the sky plane differ by $100-200 \mathrm{pc}$, that is, even for the nearest objects $1^{\prime \prime}-3^{\prime \prime}$. Therefore, to correctly distinguish the components pertaining to different subsystems, it is necessary to have high velocity accuracy $\left(10-30 \mathrm{~km} \mathrm{~s}^{-1}\right)$ and high angular resolution ( $<1^{\prime \prime}$ ). The Multi-Pupil Spectrograph (MPS), installed on the 6-m telescope in 1990, allows to achieve these resolutions (Afanasiev et al., 1990).

We present below the results of kinematical investigation of central regions in the Seyfert galaxies NGC 4151 and Mkn 573 with MPS.

\section{Results of Observations}

\subsection{NGC 4151}

The spectra were obtained with the Multi-Pupil Fiber Spectrograph in the spectral region $\lambda \lambda 4600-5300 \AA$ with a dispersion of $1.8 \AA \mathrm{px}^{-1}$ with a matrix of $8 \times 12$ spatial elements, the size of each being $0.8^{\prime \prime} \times 0.8^{\prime \prime}$. As detector we used a $2 \mathrm{D}$ photon-counting system.

The maps of the velocity field and the surface brightness distributions in the emission lines [OIII] $\lambda 5007$ and $\mathrm{H}_{\beta}$ were built (Afanasiev and Shapovalova, 1993). In [OIII] line the isophotes are elongated in the position angle $P . A . \approx 40^{\circ}$ and in $\mathrm{H}_{\beta}$ line - in P.A. $\approx 50^{\circ}$. The velocity field difference ([OIII]-H $\mathrm{H}_{\beta}$ ) gives velocity peculiarities in P.A. $\approx 15^{\circ}$, which are $\pm 100 \mathrm{~km} \mathrm{~s}^{-1}$ at distances of $100-150 \mathrm{pc}$ 
from the nucleus. It indicates slower rotation of [OIII]-emitting gas than that of the gas emitting $\mathrm{H}_{\beta}$ line. It cannot be caused by the linear radio structure detected in Booler et al.(1982) in a position angle of $77^{\circ}$. This kinematical effect may be associated with the presence of the gaseous bar in the center of NGC 4151 (Shlosman et al.,1990).

\subsection{Mkn 573}

The spectroscopic observations of the central part of Mkn 573 were carried out with MPS in the spectral regions $\lambda \lambda 4500-5500 \AA, 6000-7000 \AA$ with a dispersion of $1.8 \AA \mathrm{px}^{-1}$. An array of $9 \times 11$ square micro-lenses was used. The scale of the image constructed by one lens was $1.2^{\prime \prime} \times 1.2^{\prime \prime} .99$ spectra were recorded with the 2D photon-counting system.

From the spectra we performed a Gauss-analysis of [OIII] $\lambda 5007 \mathrm{H}_{\alpha}$ and [NII] $\lambda 6584$ emission line profiles. [OIII] line is well approximated by two gaussians, corresponding to the broad $(F W H M \approx 14 \AA$ ) and narrow components, and $\mathrm{H}_{\alpha}$ and [NII] - by one gaussian, corresponding to the narrow component.

Fig.la shows the maps of brightness distribution in the narrow component [OIII] and continuum (central wavelength $5110 \AA$, spectral window $30 \AA$ ). The isophotes in the narrow component of [OIII] are elongated in P.A. $\approx 106^{\circ}$ and coincide with the [OIII] structure observed by Afanasiev and Sil'chenko (1991).

At a distance of $2^{\prime \prime}$ from the center two knots are seen. The S-E knot is brighter than the N-W knot. The center of the narrow [OIII] component is displaced by $1.5^{\prime \prime}$ towards $\mathrm{N}$-E with respect to the continuum center.

On Fig.1b we have superimposed the narrow [OIII] component image and its velocity field. The velocity field has a regular component $\left(V_{\text {sys }} \approx 5180 \mathrm{~km}\right.$ $\mathrm{s}^{-1}$ ) in the center with a dynamical line of nodes in P.A. $\approx 66^{\circ}$. This direction is close to the photometric major axis of the disk $\left(P . A . \approx 50^{\circ}\right.$ ), determined by us from observations with a scanning Fabry-Perot interferometer of the 6-m telescope.

The isovelocity lines are strongly distorted along the [OIII] image near the knots. This indicates the presence of a noncircular component in the radial velocity. Noncircular motions in the narrow [OIII] component are $+150 \mathrm{~km}$ $\mathrm{s}^{-1}$ in S-E direction and $-150 \mathrm{~km} \mathrm{~s}^{-1}$ in N-W. This noncircular component is associated either with the radial fluxes along the cone of ionization in P.A. $\approx 106^{\circ}$, or with motions along the bar, if we associate the elongation in [OIII] with the presence of the bar-formation.

The offset of the narrow component center relative to the continuum center gives strong evidence for the presence of a bar in the central region of Mkn 573 .

On Fig.1c we have superimposed the broad component image and the isophotes of the narrow component. The center of the broad component coincides with the center in continuum.

Fig.1d shows the velocity field in the broad component [OIII] line . At a distance of $1.5^{\prime \prime}$ from the center maximum deviations $\pm 200 \mathrm{~km} \mathrm{~s}^{-1}$ of the broad radial velocity component from the systemic velocity are observed. These deviations are elongated in P.A. $\approx 125^{\circ}$ and coincide with the direction of the VLA linear radiostructure, i.e. with the direction of the radio-jet (Ulvestad et al., 1984). 
The elongation of images in [NII] $\lambda 6584$ and $\mathrm{H}_{\alpha}$ lines coincides in position angle with the elongation in [OIII] line. The velocity field in $\mathrm{H}_{\alpha}$ and [NII] corresponds to that in the narrow [OIII] line. At a distance of $4^{\prime \prime}$ from the center, in S-E direction, a bright knot in [NII] and $\mathrm{H}_{\alpha}$ lines is well seen. This knot is outside the MPS field in [OIII] line. Tsvetanov et al.(1989) noted that in this region a maximum value of $[\mathrm{OIII}] / \mathrm{H}_{\alpha}$ is reached, i.e. shock exitation is possible.

Fig. 2 shows results of observations Mkn 573 on the 6-m telescope with a scanning Fabry-Perot interferometer in [NII] 26584.

There is strong evidence that Mkn 573 is barred spiral of type SBa-SBb.

Thus, the velocity field in the central part of Mkn 573 indicates the presence of three components: 1 ) disk rotation (P.A. $\left.\approx 66^{\circ}\right)$; 2) noncircular motions along the bar or radial fluxes along the cone of ionization $\left( \pm 150 \mathrm{~km} \mathrm{~s}^{-1}\right.$, P.A. $\left.\approx 106^{\circ}\right)$; 3) radial component along the radio-jet $\left( \pm 200 \mathrm{~km} \mathrm{~s}^{-1}\right.$, P.A. $\left.\approx 125^{\circ}\right)$.

\section{Summary}

Using the Multi-Pupil Spectrograph on the 6-m telescope we can successfully study the different gas subsystems, their kinematics and ionization structures in the central regions of bright Seyfert galaxies.

\section{References}

Afanasiev V.L.,Vlasiuk V.V., Dodonov S.N., Sil'chenko O.K., 1990, Preprint SAO, No.54.

Afanasiev V.L., Sil'chenko O.K., 1991, Astrofiz.Issledovaniya (Izvestia SAO), $33,88$.

Afanasiev V.L., Shapovalova A.I., 1993 , The Nearest Active Galaxies, Ed. J.Beckman et al., Madrid.

Booler R.V., Pedlar A.A., Davies R.D., 1982 , MNRAS, 199, 229.

Shlosman I., Begelman M.C., Frank J., 1990, Nature 345, 679.

Tsvetanov Z., Tadhunter C., Perez E., Gonzales-Delgado R., 1989, in Exstranuclear Activity in Galaxies, ed. Meurs and Fosbury, 19.

Ulvestad J.S., Wilson A.S., 1984, ApJ, 278, 544. 


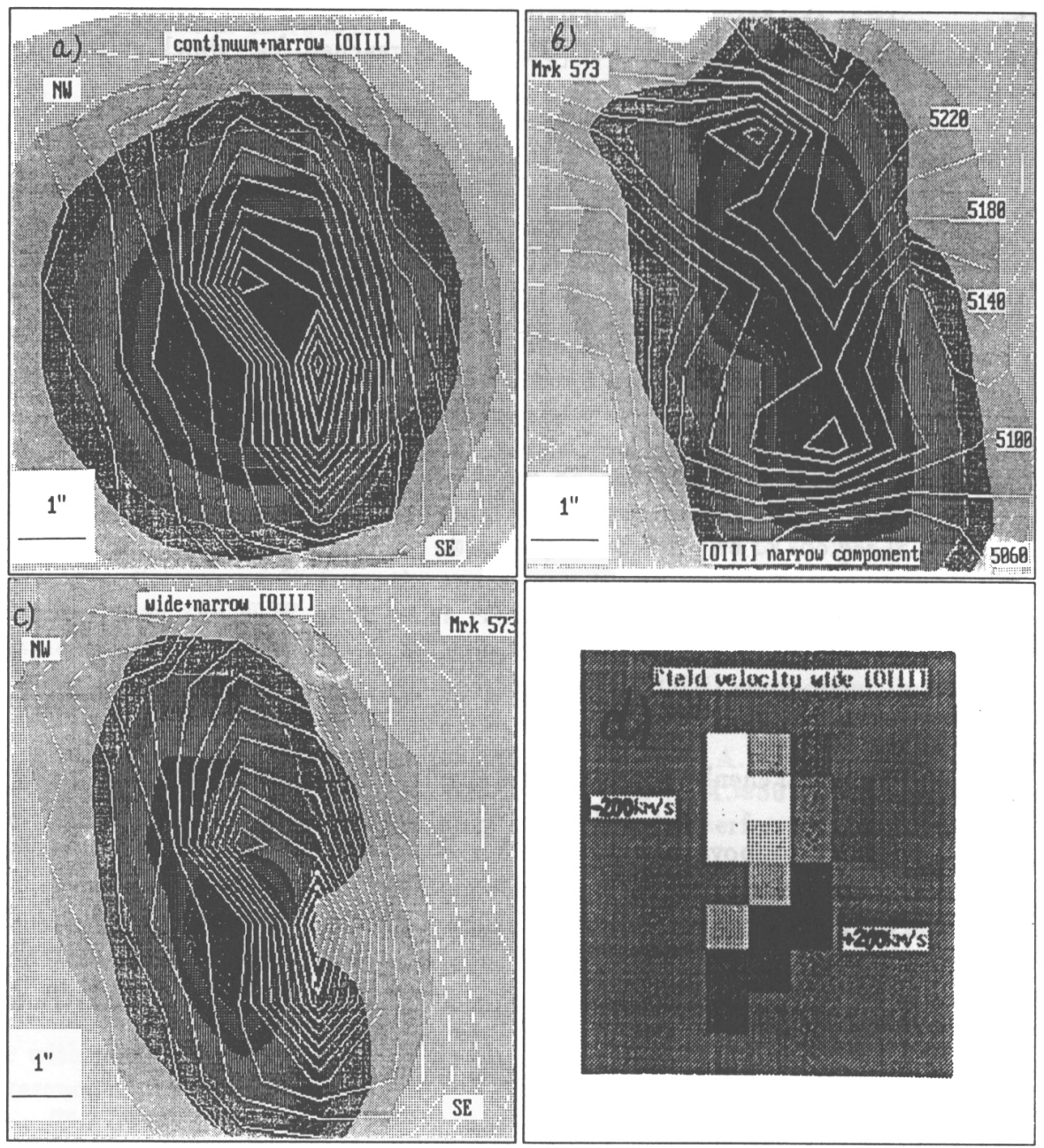

Figure 1. Markarian 573 : a - image in narrow component of [OIII] $\lambda$ 5007 (isophotes) and continuum (grey scale); b - the image in narrow component of [OIII] $\lambda 5007$ (grey scale) and its velocity field (isovelocity lines); c - image in the broad component [OIII] $\lambda$ 5007 (grey scale) and image in the narrow component [OIII] $\lambda 5007$ (isophotes); $d$ - the velocity field in broad component [OIII] $\lambda 5007$, one square is $1.2^{\prime \prime} \times 1.2^{\prime \prime}$ 


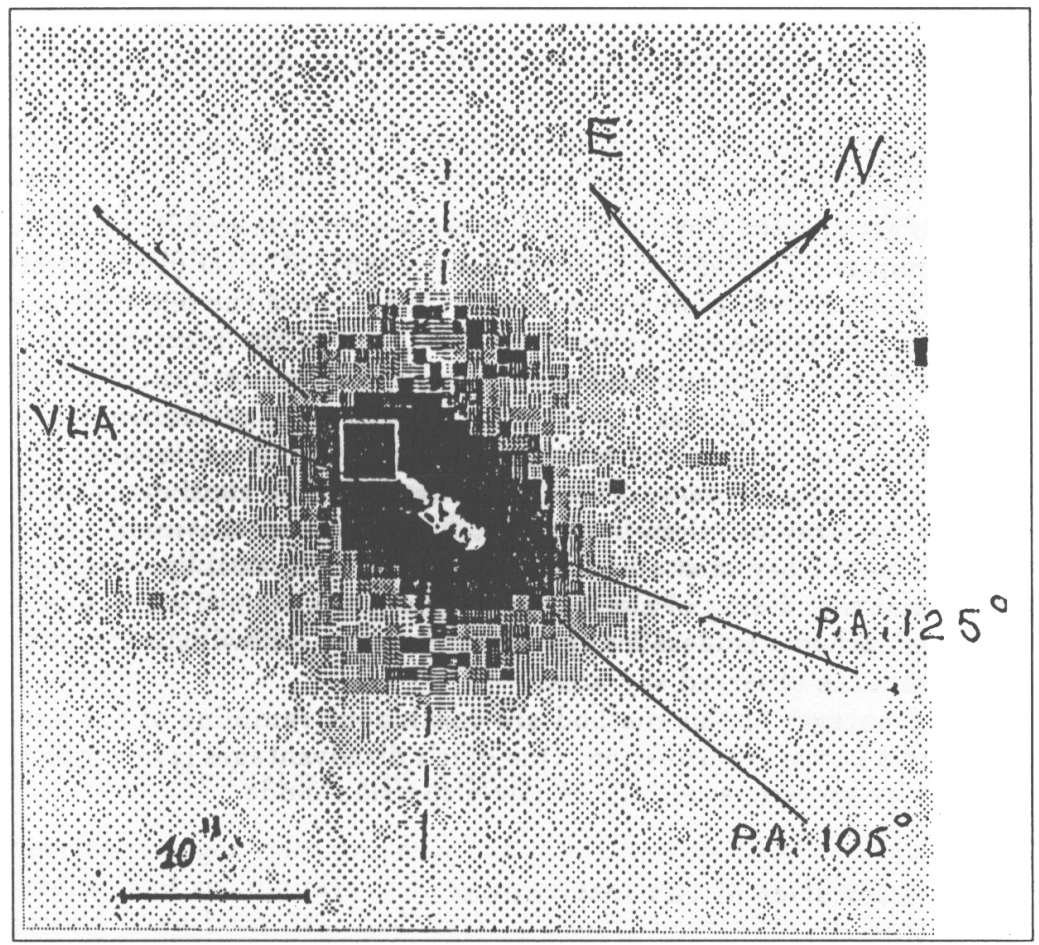

Figure 2. Mkn 573 in [NII] $\lambda 6584$ line obtained with the scanning Fabry-Perot interferometer of the 6-m telescope. 